Meta

Journal des traducteurs

Translators' Journal

\title{
Actualité internationale : les titres de presse en anglais et en français
}

\section{Ginette Demers}

Volume 39, numéro 3, septembre 1994

URI : https://id.erudit.org/iderudit/002535ar

DOI : https://doi.org/10.7202/002535ar

Aller au sommaire du numéro

Éditeur(s)

Les Presses de l'Université de Montréal

ISSN

0026-0452 (imprimé)

1492-1421 (numérique)

Découvrir la revue

Citer cette note

Demers, G. (1994). Actualité internationale : les titres de presse en anglais et en français. Meta, 39(3), 520-529. https://doi.org/10.7202/002535ar d'utilisation que vous pouvez consulter en ligne.

https://apropos.erudit.org/fr/usagers/politique-dutilisation/ 
ACTUALITÉ INTERNATIONALE: LES TITRES DE PRESSE EN ANGLAIS ET EN FRANÇAIS

Selon Haas, le journalisme attache une telle importance aux titres de presse que, «dans les grands journaux d'information, les titres ne sont pas rédigés par les rédacteurs de la copie, mais par des spécialistes» (1958: 219). Le titre, en effet, est ce qui attire en tout premier lieu l'attention du lecteur : c'est «une «vitrine» qui fait «vendre» l'article» (Agnès et Croissandeau 1979 : 47). Pourtant, les études linguistiques sur les titres de presse sont peu nombreuses, du moins en ce qui a trait aux recherches en anglais ou en français, ce sujet ayant surtout intéressé les Allemands (Hoek $1981:$ 14). Le présent article vise, à partir de l'observation d'un corpus, à dégager les similitudes et les différences stylistiques entre les titres qui figurent dans des journaux en langue française et ceux qui apparaissent dans des journaux en langue anglaise.

\section{CORPUS}

Le domaine retenu est celui de 1 'actualité internationale, la seule raison motivant ce choix étant un certain intérêt personnel pour cette sphère de l'actualité.

Le corpus se compose de 682 titres relevés dans les quotidiens suivants : Le Devoir, La Presse, Le Monde, The Gazette, The Globe and Mail et The Times.

Ces quotidiens ont été choisis parce que ce sont des journaux «d'informations générales» à vocation nationale ou provinciale, par opposition aux journaux 
régionaux qui exploitent «l'evénement spectaculaire» et aux joumaux populaires qui traitent «essentiellement le fait divers, l'information à caractère volontiers scandaleux et l'événement de type sentimental» (Bernard 1977 : 377). Ils ont été sélectionnés parmi d'autres quotidiens faisant partie de la même catégorie pour de simples raisons d'accessibilité. La décision d'inclure deux journaux européens repose sur le fait qu'il pouvait exister des différences stylistiques entre les journaux canadiens et les journaux français ou britanniques.

La periode d'observation porte sur huit jours de publication, déterminés de façon aléatoire. Seuls les titres principaux ont été étudiés en détail, les titres secondaires - sur-titres et sous-titres - n'étant traités qu'accessoirement.

Le tableau 1 illustre de façon détaillée le nombre de titres par journal de même que les dates de publication.

On remarque que le nombre de titres varie considérablement d'un quotidien à l'autre et que les titres sont plus nombreux dans les journaux de langue anglaise (379 comparativement à 303, pour les journaux de langue française, ce qui représente un écart de $25 \%$ ). Il n'est cependant pas possible de tirer des conclusions à partir d'un corpus aussi restreint: il faudrait observer davantage de journaux ainsi qu'un nombre supérieur de jours de publication pour pouvoir dégager des tendances.

\section{LES TITRES SONT-ILS TRADUITS ?}

Quoique certains articles français soient visiblement traduits de l'anglais et vice-versa et bien que certains aussi soient largement inspirés de textes figurant dans un autre journal rédigé dans la même langue, un nombre négligeable de titres semblent traduits littéralement ou empruntés directement, comme en témoignent ces exemples de titres d'articles portant sur un même sujet :

Deng s'en va mais tire toujours les ficelles (Dev 89-11-10-7) 1

Deng Xiaoping démissionne (Pre 89-11-10-A16)

$M$. Deng Xiaoping abandonne ses dernières fonctions (Mon 89-11-10-1)

Deng quits last official Communist Party post (Gaz 89-11-10-A13)

Deng quits but keeps his hold (Tim 89-11-10-1)

Provocation antisémite en France (Dev 90-0511-5)

Profanation d'un cimetière juif en France : état de choc et d' horreur (Pre 90-05-11-A10)

French leaders condemn attack on Jewish tombs ( $G a z$ 90-05-12-A13)

France searches soul after Jewish graves defiled (Glo 90-05-12-1)

Vandals despoil Jewish graves (Tim 90-05-11-1)

Pekin libère 211 opposants (Dev 90-05-11-5)

Apaisement en Chine: 211 opposants libérés (Pre 90-05-11-A10)

Libération de 211 dissidents $d u$ eprintemps de Pékin» (Mon 90-05-11-6)

China frees 211 linked to democracy drive ( $\mathrm{Gaz}$ 90-05-11-A8)

\begin{tabular}{|c|c|c|}
\hline Journaux & Dates & Nombre de titres \\
\hline Le Devoir & $\begin{array}{l}\text { 10-11-13 novembre } 1989 * \\
9-10-11-12 \text { mai } 1990\end{array}$ & 98 \\
\hline La Presse & $\begin{array}{l}\text { 10-11-12-13 novembre } 1989 \\
9-10-11-12 \text { mai } 1990\end{array}$ & 74 \\
\hline Le Monde & $\begin{array}{l}\text { 10-11-[12-13] novembre } 1989^{* * *} \\
9-10-11-12 \text { mai } 1990\end{array}$ & 131 \\
\hline The Gazette & $\begin{array}{l}\text { 10-11-12-13 novembre } 1989 \\
9-10-11-12 \text { mai } 1990\end{array}$ & 168 \\
\hline The Globe and Mail & $\begin{array}{l}\text { 10-11-13 novembre } 1989 * \\
9-10-11-12 \text { mai } 1990\end{array}$ & 75 \\
\hline The Times & $\begin{array}{l}\text { 10-11-13 novembre } 1989 * \\
9-10-11-12 \text { mai } 1990\end{array}$ & 136 \\
\hline
\end{tabular}

\section{Tableau 1}


Intellectuals among 211 jailed protesters released by China after 'lenient treatment' (Glo 90-0511-A1)

Ce fait tend à confirmer l'opinion selon laquelle le titre est «autonome par rapport au texte, [tout en formant] une unité étroite avec le texte» (Hoek 1981 : 2).

\section{POINTS ÉTUDIÉS}

Bien des auteurs estiment que «le style du titre est elliptique et nominal» (Hoek 1981:2). D'autres ajoutent que les titres se caractérisent par un usage particulier de certains signes de ponctuation ( $v$. Groupe Mu 1970: 87-88; Loffler-Laurian 1975: 117). On croit généralement, enfin, qu'un titre doit être bref et frappant. Ces constatations nous ont incitée à examiner la longueur des titres ainsi que la proportion de phrases complètes et de phrases elliptiques; sur le plan de la syntaxe, il nous a aussi semblé intéressant de relever les caractéristiques des sujets et des verbes. Pour ce qui est du lexique, la fréquence des parties du discours a été étudiée de même que celle des mots objectifs et des mots subjectifs, des images et des ambiguïtés. La ponctuation constitue le dernier point de l'analyse.

\section{SYNTAXE}

Longueur des titres

Bien qu'on considère qu'un bon titre de presse doit être «court, précis, nerveux» (Agnès et Croissandeau $1979: 47$ ), les titres observés sont longs dans l'ensemble, le nombre moyen de mots par titre se situant à 8 en français et à 7 en anglais. L'écart est essentiellement attribuable au fait qu'en anglais, les articles peuvent souvent être omis sans entorse à la syntaxe, ce qui est rarement le cas en français (voir plus loin). La marge observée est donc le résultat d'une servitude linguistique plutôt que d'un libre choix du rédacteur.

Contrairement à la tendance dégagée par Hoek pour les titres de romans contemporains (1981: 13), la proportion de titres se limitant à un, deux ou trois mots est minime (à peine $2 \%$ ):

Phnom Penh négociera (Dev 89-11-10-5)

Heseltine défie Thatcher (Dev 90-05-11-5)

Deng Xiaoping démissionne (Pre 89-11-10-A16)

Patate chaude afghane (Pre 90-05-9-A16)

Effondrement (Mon 89-11-11-1)

89 bis (Mon 89-11-11-1)

Les dominos (Mon 89-11-12-13)

Crash kills four (Gaz 89-11-11-F7)

War zone (Gaz 90-05-12-B6)

Jamaica planning GST (Glo 90-05-12-A3)

Victory for democracy (Time 89-11-11-11)

Intifada smouldering on (Time 90-05-10-13)

La longueur relativement importante des titres de presse relatifs à l'actualité internationale provient sans doute du fait qu'ils sont plutôt informatifs qu'incitatifs, c'est-à-dire qu'ils «donnent des éléments d'information en répondant à plusieurs des «questions de référence» (qui, quoi, où, quand, comment)» (Agnès et Croissandeau 1979:47) au lieu de susciter l'intérêt du lecteur par un mot ou une brève expression dont l'ambiguité n'est levée que par la lecture de l'article.

\section{Phrases complètes}

Ont été considérées comme des phrases complètes, les phrases renfermant un verbe conjugué et ne présentant aucune ellipse quant aux autres parties du discours.

Dans les journaux rédigés en français, les phrases complètes prédominent dans des proportions de $53 \%$ (Le Monde), $55 \%$ (La Presse) et $64 \%$ (Le Devoir), alors que dans les quotidiens en langue anglaise, les taux ne dépassent pas $25 \%$ (The Globe and Mail), $35 \%$ (The Times) et $45 \%$ (The Gazette). Comme pour la longueur des titres, c'est surtout l'ellipse des articles qui explique l'écart enregistré. Quant aux variations à l'intérieur d'une même langue, elles sont beaucoup moins prononcées en français qu'en anglais, le rédacteur étant soumis à plus de contraintes pour ce qui est de l'ellipse.

\section{Phrases simples/complexes}

Quoiqu'une forte proportion des phrases soient constituées d'une seule proposition, le pourcentage relativement élevé de phrases complexes - près de $25 \%$ - étonne dans un corpus composé de titres.

Dans les deux langues, ces phrases comprennent généralement une principale et une infinitive :

Papandreou est chargé de former un gouvernement (Dev 89-11-11-A6)

Gorbatchev veut réformer l'Armée rouge (Pre 90-05-9-C8)

Washington et Londres sont contraints de modi-

fier leurs positions sur le conflit (Mon 89-11-[1213]-16)

Britain finds it hard to reject isolation ( $\mathrm{Gaz} 89$. 11-13-B3)

Romanian President rejects protesters' call to delay elections (Glo 90-05-10-A12)

Kohl urges Germany to strive for unity (Tim 89. 11-11-3)

Exceptionnellement, les phrases renferment trois propositions et même, dans un cas, quatre :

L'Ouest se réjouit, l'URSS approuve, mais met en garde (Dev 89-11-11-A5)

$M$. Deng Xiaoping affirme qu'il continuera $\grave{a}$ «jouer son rôle comme il se doit" (Mon 89-1111-8)

M. Najabullah se dit prêt à abandonner le pouvoir s'il perd aux élections (Mon 90-05-9-4)

They came, saw and they drank (Gaz 89-11. 11-A4)

Moscow signals it would allow Berlin communists to fall (Tim 89-11-10-8) 
Sujets

Pratiquement tous les sujets sont à la troisième personne. Comme le fait remarquer Turner à la suite de l'observation d'un corpus constitué d'une centaine de titres extraits de journaux australiens, une proportion très élevée d'entre eux désignent des animés humains, «either a name, a word referring to the holder of a rank or a word referring to a group of human agents such as subcommittee or Indonesia (considered as a government)» (1972: 73). Pour ce qui est de notre corpus, le taux atteint $88 \%$, tant en anglais qu'en français. Les noms propres - noms de personnes, de peuples, d'organisme, noms de villes ou de pays désignant un gouvernement - sont nettement prééminents, mais il y a aussi un petit pourcentage de noms communs. Voici quelques exemples :

Deux candidats à la présidentielle veulent se retirer (Dev 90-05-10-5)

Les travaillistes israéliens étalent leurs divisions (Pre 90-05-10-A16)

Les nouveaux dirigeants de la RDA promettent une démocratisation (Mon 89-11-10-1)

Octogenarians square off again (Gaz 90-05-10A9)Jubilant citizens head West (Glo 89-11-10-1)

Visitors besiege Western stores (Tim 89-11-13-10)

Les sujets désignant des êtres inanimés, quant à eux, sont souvent des noms abstraits :

L'appartenance au Pacte de Varsovie est maintenant une question ouverte (Dev 90-05-12-A7)

La folie «sage» continue (Pre 89-11-12-A1)

Pour Bonn, «le socialisme touche à sa fin...» (Mon 89-11-11-5)

Violence in Moldavia leaves 129 injured ( $G a z$ 89-11-12-B8)

Democracy in India stops short of Punjab (Glo 90-05-10-A7)

Pressure increases on Peres (Tim 90-05-10-A7)
Verbes

À l'exception d'un impératif et d'un conditionnel, les verbes des propositions indépendantes ou principales sont tous au mode indicatif. L'emploi du conditionnel, ressource qui s'offre au journaliste lorsqu'il veut présenter l'événement comme une simple éventualité ou encore quand il ne veut pas prendre à son compte ce qu'il annonce, semble donc beaucoup moins fréquent qu'on le suppose couramment.

Les verbes sont au présent dans des proportions moyennes se situant à $82 \%$ en français et atteignant $98 \%$ en anglais. Le tableau 2 montre un écart assez important entre Le Devoir, La Presse et Le Monde, alors que les taux sont très homogènes dans les journaux rédigés en anglais.

Il reste que, comparativement au passé et au futur. le présent prédomine largement dans les six quotidiens : «pour le journaliste, il s'agit de conserver à la nouvelle sa fraîcheur première» (Groupe Mu 1970: 86) et de donner ainsi au lecteur l'impression qu'il le met immédiatement au courant de ce qui s'est passé. Le présent est sans contredit le temps de l'actualité.

Les verbes au passé sont soit au passé composé, soit au simple past. L'emploi de ces temps indique, le plus souvent, qu'on a parlé de l'événement dans les jours précédents, comme l'illustrent les exemples suivants :

Berlin-Est ouvre le Mur (Pre 89-11-10-A1)

55000 Allemands de l'Est ont franchi la frontière ces dernières 24 heures (Pre 89-11-11-F10)

Les ministres de la défense amorcent la réflexion sur la nouvelle stratégie de l'OTAN (Mon 90-0510-4)

Les ministres de la défense ont amorcé la réflexion sur une nouvelle stratégie de l'OTAN (Mon 90.

05-12-4)

Berliners rejoice as Wall opens (Gaz 89-11-10-A1)

They came, saw and they drank (Gaz 89-1111-A4)

Temps des verbes $(\%)$

\begin{tabular}{lcccc}
\hline Journaux & Présent & Passé & Futur & Total \\
\hline Dev & 92 & 2 & 6 & 100 \\
Pre & 71 & 17 & 12 & 100 \\
Mon & 80 & 14 & 6 & 100 \\
Gaz & 94 & 6 & 0 & 100 \\
Glo & 100 & 0 & 0 & 100 \\
Tim & 98 & 0 & 2 & 100 \\
\hline
\end{tabular}

Tableau 2 
Il arrive, cependant, qu'on ait recours au passé pour insister sur l'aspect de finalité d'une nouvelle :

L'armée rouge a coûté un milliard à la Pologne (Dev 90-05-10-5)

Ochoa a été fusillé pour conspiration contre Castro (Pre 89-11-10-A16)

Les premières négociations entre le président

lliescu et les contestataires ont tourné court (Mon 90-05-9-A8)

'God turned his face away' (Gaz 90-05-9-A8)

Le futur, enfin, indique la simple postériorité d'un événement par rapport au moment où le journaliste parle :

Phnom Penh négociera (Dev 89-11-10-5)

Le.problème des sanctions contre Pretoria sera abordé par les Douze à Dublin en juin (Pre 9005-11-A10)

Le sommet arabe se tiendra à Badgad (Mon 90 05-12-6)

The Curtain lifts : will the cast stay? (Tim 89-1110-16)

Remarquons qu'en français, le futur est tout aussi fréquemment exprimé par le semi-auxiliaire aller suivi de l'infinitif que par le futur simple:

L'engagement canadien va devenir déterminant (Dev 89-11-10-5)

L'État brésilien va licencier un quart des fonctionnaires (Mon 90-05-11-1)

Des représentants de la CEE vont rencontrer une délégation iranienne à Dublin (Mon 90-05-12-4)

Notons également qu'en anglais, il n'y a que deux titres où le futur est employé dans une phrase complète. L'anglais, en effet, semble préférer une forme elliptique : be to suivi de l'infinitif avec suppression du mot be:

Bush, Shamir to hold meeting next week ( $G a z$ 8911-10-A14)

Hungary to consider proposal to quit Warsaw Pact (Glo 90-05-10-A12)
Soviet 'democracy' to stay under control of party (Tim 89-11-13-11)

$E C$ and Iran to discuss hostages in Dublin talks (Tim 90-05-11-10)

La voix active est privilégiée, le pourcentage de titres où figurent des verbes au passif se limitant à $6 \%$ en français et à $13 \%$ en anglais. Le pronom on est inexistant dans le corpus observé; l'emploi du passif en français s'explique essentiellement par la réticence du rédacteur à avoir recours à ce pronom :

Papandreou est chargé de former un gouvernement (Dev 89-11-11-A6)

Ochoa a été fusillé pour conspiration contre Castro (Pre 89-11-10-A16)

$M$. Yildirim Akbulut a été nommé chef du gou* vernement (Mon 89-11-11-6)

L'anglais préfère le passif à la forme one suivie d'un verbe à l'actif, mais il arrive aussi que le passif soit accompagné d'un complément d'agent. Dans ce cas, le français fait plutôt appel à la voix active :

L'Albanie humanise sa justice et autorise la pratique religieuse (Pre 90-05-10-A15)

Albanie Le Parlement a valé plusieurs mesures de libéralisation (Mon 90-05-11-3)

Human rights reform policy announced by Albania (Glo 90-05-10-1)

Ellipses

Les différents types d'ellipses ainsi que les taux par journal figurent au tableau 3 .

Mentionnons d'abord que le pourcentage élevé d'ellipses de prépositions dans La Presse est attribuable au fait que le lieu où se produit l'événement est intégré au titre — où il est mis en relief pas les deux points - alors que, dans les autres quotidiens, il figure pratiquement toujours en sur-titre :

Haiti : arrestations dénoncées (Pre 89-11-11-F10) Namibie: les principaux partis satisfaits (Pre 89 11-13-A13)

Pologne: les étudiants font la grève (Pre 90-0510-A15)

Types d'ellipses (\%)

\begin{tabular}{lccccc}
\hline Journaux & Sujet + verbe & Verbe & Articles & Prépositions & Total \\
\hline Dev & 50 & 19 & 31 & 0 & 100 \\
Pre & 25 & 21 & 31 & 23 & 100 \\
Mon & 42 & 16 & 39 & 3 & 100 \\
Gaz & 6 & 34 & 56 & 1 & 100 \\
Glo & 3 & 41 & 48 & 0 & 100 \\
Tim & 16 & 36 & & 0 & 100 \\
\hline
\end{tabular}


Dans les journaux de langue française, c'est l'ellipse sujet + verbe qui prédomine, tandis que, dans les quotidiens de langue anglaise, elle est plutôt rare. Precisons que ce type d'ellipse n'est possible que s'il $s$ 'agit de tours impersonnels tels il y $a$, there is :

Coupes sombres au Pentagone (Dev 89-11-10-5) Accord de principe entre Téhéran et Washington (Pre 90-05-10-A14)

Libération de deux cent onze dissidents du "printemps de Pékin" (Mon 90-05-11-6)

Dancing, fireworks and cries of 'Come on over' (Gaz 89-11-11-1)

Crisis in way of life in Korea despite three years of democratic rule (Glo 90-05-12-1)

Grief at the loss of nerve (Tim 89-11-11-A4)

Notons également que le français n'admet l'omission de l'article que dans le cas du premier nom de ce genre d'énoncé ou encore dans le cas d'un nom mis en évidence par les deux points :

Réunification: James Baker prône la prudence (Pre 89-11-13-A12)

L'anglais est beaucoup plus libéral, comme le montrent les exemples suivants :

Construction of controversial temple halted by Indian judge ( $G a z$ 89-11-13-B8)

Abortion pill 'chimical bomb' - Vatican paper (Gaz 89-11-13-F9)

Soup kitchen solace for Kadar's victims (Tim 90 05-9-12)

En outre, l'ellipse du verbe - y compris celle des auxiliaires - est plus fréquente dans cette langue. Le verbe to be, en particulier; est supprimé trois fois plus souvent que l'est le verbe être. À la suite de l'observation de son corpus de titres de presse, Turner a lui aussi remarqué que les «Determiners and the verb to be are almost universally omitted» (1972: 72). En raison de ces ellipses systématiques, les titres anglais sont bien davantage que les titres français «le reflet du 'style télégraphique' des dépêches d'agence» (Groupe Mu 1970: 86).

\section{LEXIQUE}

\section{Fréquence des parties du discours}

Les mots-outils ont été regroupés. En effet, dans les deux langues, la fréquence des pronoms et des conjonctions est très faible. En français, l'emploi des mots-outils se limite essentiellement aux prépositions et aux articles, tandis qu'en anglais, il se limite en général aux adjectifs déterminatifs et aux prépositions.

Les pourcentages sont très uniformes d'un journal à l'autre. Le tableau 4 montre les taux moyens pour le français et pour l'anglais.

On voit que la fréquence des adverbes est négligeable. Dans les quelques titres où ils apparaissent, ils ont le plus souvent une valeur affective :

Les Allemands se déplacent désormais librement de l'Est à l'Ouest (Mon 89-11-11-1)

Jean-Paul II dénonce vigoureusement l'injustice sociale et la corruption (Mon 90-05-10-7)

Astonished East Germans ask: 'Is it really true?' (Gaz 89-11-10-A10)

Nato's chief wary of change too quickly (Glo 8911-10-A4)

Rocard says $U K$ was 'too hasty' when joining $E C$ (Tim 89-11-10-9)

On constate également que la proportion d'adjectifs qualificatifs est peu élevée. Il s'agit, en genéral, d'adjectifs dénotatifs. Environ $35 \%$ d'entre eux, cependant, sont connotatifs. Quelques exemples illustrent les deux cas :

En échange d' un statut, l'Église mexicaine ne fera pas de politique, promet le pape (Dev 90-05-9-4) Les travaillistes israéliens étalent leurs divisions (Pre 90-05-10-A16)

L'idée d' une conférence euro-arabe n'enthousiasme pas les partenaires européens (Mon 89. 11-10-7)

Bulgarian leader swept away by tide of change (Gaz 89-11-11-B1)

Soviets dismiss talk of German reunification (Glo 89-11-11-A6)

French right wing under attack after graves attack (Tim 90-05-12-9)

Fréquence des parties du discours (\%)

\begin{tabular}{lcccccc}
\hline Journaux & Noms & Verbes & $\begin{array}{c}\text { Adjectifs } \\
\text { qualificatifs }\end{array}$ & Adverbes & Mots-outils & Total \\
\hline Dev-Pre-Mon & 37 & 13 & 7 & 0 & 43 & 100 \\
Gaz-Glo-Tim & 48 & 17 & 7 & 0 & 28 & 100 \\
\hline
\end{tabular}


Raz-de-marée euphorique à Berlin (Dev 89-1113-1)

Situation de plus en plus tendue en Roumanie (Pre 90-05-12-G9)

Spectaculaire recrudescence de la délinquance (Mon 89-11-10-6)

Gorbachev attempts to bolster sagging morale of the military ( $\mathrm{Gaz}$ 90-05-9-A8)

Jubilant citizens head West (Glo 89-11-10-A1)

Dirty tricks surface in Balkan style election (Tim 90-05-9-12)

En anglais comme en français, la proportion de verbes est près de trois fois inférieure à celle des substantifs. Par contre, le taux de verbes affectifs est trois fois plus élevé, les verbes «neutres» (être, il y $a$, to be, there is, etc.) étant souvent omis, surtout en anglais. Voici certains des titres où figurent des verbes à valeur connotative :

L'Amérique aime Corazon Aquino (Dev 89-11. 10-07)

La direction de l'intifada demande au sommet arabe de dénoncer les États-Unis (Pre 90-0512-G9)

$M$. Bush applaudit, mais avec retenue (Mon 8911-11-40)

Reform economy fast, warns East Germany ( $G a z$ 90-05-11-1)

Speed of turnabout startles even Soviets (Glo 8911-13-A9)

Comecon chief pleads for time (Tim 90-05-11-8)

Parmi les mots pleins «le substantif accompagné ou non de l'article [...] a la vedette» (Grivel 1973: 167). Les noms propres sont omniprésents : il suffit, en effet, de se reporter aux titres cités plus haut pour constater qu'à de rares exceptions près, il y en a au moins un par titre. Dans tous les journaux observés, les substantifs sont objectifs à plus de $90 \%$. Ils servent ordinairement à identifier des personnalités politiques, des pays, des villes, des organismes, des partis politiques, etc.

$\mathrm{Au}$ nombre des titres où figurent des noms subjectifs, on trouve:

L'Afrique du Sud n'est pas une pestiférée (Dev 90-05-9-5)

Profanation d'un cimetière juif en France: état de choc et d' horreur (Pre 90-05-11-A10)

Tonnerre d'applaudissements au Bundestag (Mon 89-11-11-4)

Northern Ireland's days and nights of terror ( $\mathrm{Gaz}$ 90-05-12-B6)

Threat of religious bloodbath clouds India election campaign (Glo 89-11-13-A9)

Japan loses patience with Kremlin foot-dragging (Tim 89-11-13-11)

Il reste que l'ensemble du vocabulaire utilisé dans les titres de presse relatifs à l'actualité internationale est bien plus souvent dénotatif que connotatif, ce qui confirme l'hypothèse formulee dans le paragraphe sur la longueur des titres, selon laquelle ces titres sont plutôt informatifs qu'incitatifs.

Si l'on se reporte au tableau 4, on constate que la proportion de substantifs est plus importante dans les titres anglais que dans les titres français, le pourcentage de mots-outils étant inférieur. Lorsque le calcul est effectué pour les seuls mots pleins, le taux devient identique $(66 \%)$. Ce résultat tend à infirmer l'opinion très répandue voulant que le français, contrairement à l'anglais, privilégie le substantif (v. Vinay et Darbelnet 1977 : 102-104; Delisle 1980 : 177).

Images

Le rédacteur de titres de presse a rarement recours aux images: elles n'apparaissent que dans $3 \%$ des titres, dont :

L'Europe tire le rideau, 45 ans plus tard (Dev 90-05-9-5)

L'islamisme frappe aux portes de la Chine (Dev 90-05-10-A7)

Les dominos (Mon 89-11-[12-13]-1)

Mosaïque canadienne (Mon 90-05-9-1)

Artistic revival flowers after drought of oppression (Gaz 89-11-12-B9)

Experts say AIDS virus may have accomplice (Glo 90-05-11-A4)

Perestroika bites the dust (Glo 90-05-11-A7)

Kohl treads delicate diplomatic tightrope in Poland (Tim 89-11-10-8)

Nato papers over the cracks (Tim 90-05-11-8)

Ambiguiltés

Le nombre de titre dont l'ambiguïté ne peut être levée que par la lecture de l'article est minime :

Quatre observateurs du Canada (Dev 90-0512-A5)

Patate chaude afghane (Pre 90-05-10-A16)

Effondrement (Mon 89-11-11-1)

89 bis (Mon 89-11-11-1)

Les dominos (Mon 89-11-[12-13]-1)

'God turned his face away' (Gaz 90-05-9-A8)

En effet, bien que les titres secondaires (sur-titres et sous-titres) accompagnent souvent des titres principaux ne présentant aucune ambiguitté - dans ce cas, leur rôle est de fournir un simple supplément d'information - il arrive qu'ils soient explicatifs et qu'ils permettent de comprendre immédiatement un titre principal qui, autrement, ne serait pas clair. Voici quelques exemples:

Aux négociations sur le désarmement (sur-titre) Pologne et Hongrie en solo (Dev 89-11-10-7) Les Berlinois n'en reviennent toujours pas ! (Surtitre)

La folie «sage» continue (Pre 89-11-12-A1)

RDA: les changements à la tête du parti et du gouvernement (sur-titre)

Records de vitesse (Mon 89-11-10-3) 
Octogenarians square off again

Elections set for May 16 (sous-titre) (Gaz 90-05-

10-A9)

Berlin Wall opens (sur-titre)

Jubilant citizens head West (Glo 89-11-10-A1)

Soldiers of misfortune

Cuba's troops are bringing home an unanticipated and lethal legacy of the Angolan war (sous-titre) (Tim 89-11-10-14)

\section{PONCTUATION}

Les signes de ponctuation sont peu utilisés: la proportion de titres sans ponctuation est d'environ $70 \%$ dans La Presse, Le Monde, The Gazette et The Globe and Mail et atteint quelque $90 \%$ dans $L e$ Devoir et The Times.

Le point, d'une rareté extrêtme, n'est employé qu'après un mot en abrégé, jamais à la fin d'une phrase. Le point d'exclamation, le point d'interrogation, les points de suspension, le point-virgule et le tiret figurent dans moins de $2 \%$ des titres. Les guillemets sont le signe de ponctuation le plus fréquent ( $9 \%$ des titres). Ils sont suivis par la virgule et les deux-points $(7 \%$ et $5 \%$ des titres respectivement). Les taux varient considérablement d'un journal à l'autre, cependant, de sorte qu'il est difficile d'observer des tendances (tableau 5).

Les guillemets servent très rarement à attirer l'attention sur un mot ou une expression mais sont plutôt utilisés lorsqu'une citation figure dans le titre.

La citation produit un effet d'authenticité, et c'est le journal Le Monde qui en fait le plus grand usage. Il est intéressant de faire remarquer que la proportion y est de trois à quatre fois plus élevée que dans les quotidiens canadiens rédigés en français, alors que les pourcentages sont très homogènes pour les journaux en langue anglaise. Les exemples suivants illustrent cet emploi des guillemets :
«Désarmez tous les civils blancs» demandent les Noirs d'Afrique du Sud (Dev 90-05-12-A5)

"Un pas vers l'Europe une et libre»-George Bush (Pre 89-11-10-A17)

Pour Bonn, «le socialisme touche d̀ sa fin...» (Mon 89-11-11-5)

'We will lose our jobs', guards fret as notorious Berlin crossing opens ( $G a z$ 89-11-10-1)

Reunification would be 'grave problem' for Jew's (Glo 89-11-13-A10)

UN envoy praises poll in Namibia as 'free and fair' (Tim 89-11-13-13)

La mise en relief s'effectue de préférence au moyen des deux-points, bien que ce signe de ponctuation n'apparaisse ni dans Le Devoir ni dans The Globe and Mail. Parfois, c'est le lieu où s'est produit l'événement qui est ainsi mis en évidence.

(1) Pérou: la droite triomphe aux municipales (Pre 89-11-13-A13)

(2) Amérique latine: la tentation néolibérale (Mon 89-11-11-1)

(3) Germany: a diary of change ( $G a z$ 89-11-10 A10)

Rappelons que cette ressource est systématiquement utilisée dans La Presse alors que, dans les autres quotidiens, le lieu est généralement indiqué en sur-titre.

Il arrive aussi que le rédacteur ait recours aux deux-points pour mettre le thème en relief, quoique celui-ci soit tout aussi souvent signalé en sur-titre. Voici des exemples des deux cas :

(4) Réunification: James Baker prône la prudence (Pre 89-11-13-A12)

(5) Massacre sur un campus au Pendjab: 25 morts (Mon 89 -11-11-8)

(6) The Berlin Wall: great barrier might become door to freedom (Gaz 89-11-10-A10)

Ponctuation ( $\%$ des titres)

\begin{tabular}{lccccc}
\hline Joumaux & Guillemets & Virgule & Deux-points & Autres & Total \\
\hline Dev & 6 & 7 & 0 & 0 & 11 \\
Pre & 4 & 1 & 20 & 5 & 30 \\
Mon & 18 & 3 & 4 & 3 & 28 \\
Gaz & 8 & 13 & 8 & 2 & 31 \\
Glo & 7 & 23 & 0 & 0 & 30 \\
Tim & 7 & 0 & 1 & 1 & 9 \\
\hline
\end{tabular}

\section{Tableau 5}


Événements en RDA

L'Ouest se réjouit, l'URSS approuve mais met en garde (Dev 89-11-11-A5)

Des militants communistes ont manifesté à Berlin-Est

«Faites confiance à la base» (Mon 89-11-10-3)

The refugee problem

New arrivals stretch port's resources and hospitability (Tim 89-11-13-10)

Dans quelques titres, enfin, ce signe de ponctuation permet d'attirer l'attention sur l'auteur d'une déclaration:

(7) Le pape aux patrons : si le socialisme a perdu, le capitalisme n'a pas gagné (Pre 90-05-10A13)

(8) Can't relay decision on NATO: Kohl (Gaz 90-05-9-A12)

Ces usages particuliers des deux-points constituent l'une des caractéristiques de la syntaxe des titres. En effet, «À l'ordinaire modeste signe de ponctuation, il [ce signe] acquiert ici valeur de morphème. Il signale et supplée une absence (ellipse) mais il participe aussi bien à une mise en évidence (hyperbate)» (Groupe $\mathrm{Mu} 1970: 87$ ). Notons, cependant, que la valeur des deux-points est variable: dans les exemples 1,2 et 3 , ils indiquent une complémentation circonstancielle; dans l'exemple 5, ils servent de liaison entre deux énoncés et annoncent une conséquence; dans les exemples 7 et 8 , ils suggèrent un discours direct; les exemples 4 et 6 , quant à eux, peuvent s'interpréter «soit comme une apposition thématique (indiquant le champ dans lequel situer ce qui suit, sorte de rubrique), soit comme une complémentation de type «en ce qui concerne» (Loffler-Laurian 1975 : 117).

$\mathrm{Si}$, en français, la fréquence d'emploi de la virgule est assez uniforme, il n'en est pas de même en anglais : ce signe est inexistant dans The Times alors qu'il apparaît dans $13 \%$ des titres relevés dans The Gazette et dans $23 \%$ des titres extraits du Globe and Mail.

La virgule s'utilise à la suite d'une assertion lorsque le verbe de la principale est exprimé :

Israël ne négociera pas avec l'OLP, déclare Arens (Dev 89-11-11-A6)

Le gouvernement a épuisé ses possibilités, lance Walesa (Dev 90-05-11-5)

Soviets may have fired lasers at U.S. plane, Pentagon says (Gaz 89-11-10-Al3)

Move on Lithuania, Soviet army urges (Gaz 90. 05-9-A8)

Kremlin can live with change in East Germany, spokesman declares (Glo 89-11-10-A4)

Military must embrace Perestroika, Gorbatchev says (Glo 90-05-9-A4)

Remarquons que cet emploi de la virgule est plus courant dans The Gazette et The Globe and Mail que dans les quotidiens rédigés en français. De plus, contrairement au français, l'anglais a recours à ce signe pour séparer des sujets :
Bush, Shamir to hold meeting next year (Gaz 8911-10-A14)

Pretoria, ANC learn to cooperate ( $G a z$ 90-05-9H12)

Black, white Namibians live separate lives (Glo 89-11-13-A11)

Ecalogists, Indian group set to meet (Glo 90-059-A5)

Par contre, dans les deux langues, la virgule sert à isoler un participe passé, une participiale absolue ou un complément circonstanciel placé en tête de phrase :

En échange d'un statut, l'Église mexicaine ne fera pas de politique, promet le pape (Dev 90-059-4)

Partis voir le pape, ils trouvent la mort (Dev 9005-11-4)

Les travaillistes, divisés, hésitent sur la stratégie à suivre (Mon 90-05-10-5)

For emotional reasons, many Israelis don't want Germany to be reunited (Gaz 89-11-13-B1)

Mutilated by bomb, priest recovering (Glo 90-0512-A4)

\section{CONCLUSION}

Peu de différences ont été observées entre les journaux canadiens et les journaux européens. Le Monde se distingue des autres quotidiens par l'emploi systématique des titres de civilité (M., Mme), par une utilisation plus fréquente des guillemets et par le fait que les nombres y sont inscrits en toutes lettres. Pour ce qui est du Times, la seule divergence est l'absence de virgules. Il est possible que ces particularités ne se retrouvent pas dans d'autres journaux français ou britanniques; il faudrait analyser un corpus plus vaste pour le déterminer.

Les similitudes les plus frappantes entre le français et l'anglais sont la longueur des titres, la prééminence de la déclaration, celle du temps présent et des sujets désignant des animés humains, l'omniprésence des noms propres, la nette prédominance des substantifs sur les autres parties du discours, la rareté des adjectifs qualificatifs et des adverbes, le recours à un vocabulaire dénotatif plutôt que connotatif, la quasi-absence d'images et d'ambiguîtés ainsi qu'un emploi restreint des signes de ponctuation. On peut donc dire que, dans les deux langues, les titres sont en général de type informatif et qu'ils se caractérisent par un style nominal.

Parmi les différences, soulignons que la proportion d'ellipses est supérieure dans les titres anglais. De plus, c'est l'ellipse sujet + verbe qui prédomine en français, tandis qu'en anglais, c'est celle de l'article et du verbe. Le taux de verbes au passif est également plus élevé dans les titres anglais.

En raison de la fréquence d'emploi de certaines structures syntaxiques et lexicales, en raison aussi de l'absence complète d'autres structures, la langue des titres de presse portant sur l'actualité internationale, qu'il s'agisse de l'anglais ou du français, peut être considérée comme une langue de spécialité, au sens 
où le style y est le reflet «d'un choix opéré parmi les moyens d'expression fournis par la langue en vue de répondre aux nécessités propres à une forme de communication donnée» (Vigner $1976: 26$ ).

GINETTE DEMERS

Université Laval, Québec, Canada

\section{Notes}

1. La source des exemples a été abrégée de la façon suivante: les trois premières lettres du nom du journal suivies de l'année de publication, du mois, du jour, de la section s'il y a lieu et de la page. Ainsi, «Pre 89-11-10-A16» se lit : La Presse, 10 novembre 1989 , section $A$, page 16 .

\section{RÉFÉRENCES}

AGNÈS, Yves et Jean-Michel CROISSANDEAU (1979) : Lire le journal, Saint-Julien-du-Sault, F. P. Lobies.

BERNARD, Yves (1977): «Typographie et presse quotidienne», L'espace et la lettre, Paris, Union Générale d'Édition, pp. 371-392.

DELISLE, Jean (1980): L'analyse du discours comme méthode de traduction, Ottawa, Éditions de l'Université d'Ottawa.

GRIVEL, Charles (1973): Production de l'intérêt romanesque, La Haye, Mouton.

GROUPE MU (1970): Rhétorique générale, Paris, Larousse.

HAAS, C. R. (1958) : La publicité. Théorie, technique et pratique, Paris, Dunod.

HOEK, Léo-H. (1981) : La marque du titre, La Haye, Mouton.

LOFFLER-LAURIAN, Anne-Marie (1975) : «Lexique et fonction dans les titres de presse», Cahiers de lexicologie, XXVI-1, Paris, Didier-Larousse, pp. 110-124.

TURNER, G. W. (1972) : «The Grammar of Newspaper Headlines Containing the Preposition On in the Sense 'About'», Linguistics, 87, The Hague, Mouton, pp. 71-86.

VIGNER, Gérard (1976) : «Initiation à l'expression écrite dans les langues de spécialité. L'objectivation», Le Français dans le Monde, 122, Paris, Hachette-Larousse, pp. 26-32, 41-42.

VINAY, J.-P. et Jean DARBELNET (1977) : Stylistique comparée du français et de l'anglais, Montréal, Beauchemin. 